\title{
Research on the Application of Stratification Technology in Computer Software Development
}

\author{
Weiguo Zou ${ }^{1, \text { a }}$ \\ ${ }^{1}$ Yancheng Vocational Institute of Industry Technology, Yancheng, 224001 \\ ${ }^{a}$ email
}

Keywords: Stratification Technology, Computer Software Development, Application Status

\begin{abstract}
With the development of network technology, computer software is developing from the two story structure model to a multi-layer structure. In the multilayer structure, middleware is the key layer of application layer, application software development has become a new technology, and with databases, operating systems form the basis of computer software. With the computer software application environment increasingly complex, design multi-layered architecture, use software layering and modularity that allows software process become clearer and easier to maintain and extend, enhance the flexibility of computer software development and adaptability, thus stratification technology has been widely used in modern computer software development.
\end{abstract}

\section{Introduction}

Stratification technology is a computer software development trends, mainly because stratification technology for computer software development has many advantages. Computer software was developed to meet consumer demand and produce high quality software products, and for computer software development, in order to gradually reduce working time and improve efficiency through rigorous testing can be used to create computer software development member the system is also able to ensure that the entire software developed with high performance and high quality. Software system is capable of using a layered member, and the underlying physical hardware to build relationships between the components, in order to constantly improve the performance of computer software systems. Computer software for the overall system, its software does not have a relatively tight structure of the system, having generated between the multiple layers of computer software will make the upper and lower levels of a dependency relationship, and from the analysis of a wide range of terms mainly gathered in the many levels of members, and only from the microscopic point of view, can be said that the relationship between the level of available internal hierarchy is a link. Computer software used in the development of hierarchical abstraction technique can promote the development of the software system, and constantly changing software systems will be part of the complex into the software design. And computer software development if you use layered technology it has good stability and scalability, while one of the level of change will not affect the overall change is only likely to affect the level of the upper and lower parts. At the same stratification technology also enables the computer to automatically receive software developers to use, and has a reusable functions, as long as between each level can have a unified standard interface, it will be able to ensure seamless between one kind of individual software connections combined.

\section{Stratification Technology Analysis in Software Development}

In the continuous development of computer technology in its software development level by two, three multi-layer architecture development, stratification technology has been constantly improve the development of software functions at different levels of the architecture is different, next, two, three and four-layer structure are also analyzed.

In the two computer software model which, mainly by the server and the client database constituted, in which the client can provide the user interface, and is responsible for about logic, and the server to the client's instructions to accept, and query the database according to instructions and 
returns the results of the query, such logic structure called Fat client, the client of this Layer technology implemented a number of business logic, once the number of clients increases, there will be poor scalability, no interaction, communication applications poor, large maintenance costs and the existence of security risks and other disadvantages. With the large demand for computer application system, the original two-story structure can not meet the technical requirements of the application system, the emergence of the three-tier structure technology, this level of technology mainly by the client, application server and data server configuration, which provides the client human-computer interaction, the data server can provide data access, storage and optimization, and application servers to achieve relevant business logic, effectively reducing the burden on the client, to be called thin clients, such as the software architecture of ERP systems on Hitachi using a three-tier structure technology, including an interface layer (client), business process and data layers and the like, wherein the interface layer is responsible for the user interface and the system, the user receives a software application operation; data layer is responsible to after the whole system operational data to provide, under consideration is a system of data transmission efficiency, performance and data security, and business process layer is based on the user software requirements, user instructions given analysis processing, the data layer data requested operation $\mathrm{He}$ suggested that this three-tier C/S structure to achieve the purpose of the application. Compared with the two-story structure, this stratification technology has reusable, easy maintenance, scalability, high security, and effectively reduce the network load and so on, stratification technology three-layer structure in spite of many advantages, but the two-story structure and not from the historical stage, the number of users in less time, two-layer structure has more advantages. Therefore, software development, should be based on the actual situation, decide what stratification technology.

\section{Characteristics and Significance of Stratification Technology in Software Development}

With computer software performance requirements continue to increase, the need to apply the new technology in computer software development. For example, the application of modular design, software architecture and stratification technology multi-layer design technology, to a great extent changed the performance and efficiency of computer software and computer software during the development process, increasing its adaptability and flexibility. Computer software development is mainly to improve the quality of calculation software, and constantly adapt to computer users to add new applications. With member-based computer software developing, the need for rigorous testing, after testing qualified before as a member and to build software systems are important. This procedure, during computer software development, reduced working time and work efficiency and it is to protect the new computer performance quality. Computer software can also run the system provides a common arithmetic, by grouping all levels of building blocks, the underlying database connections, components and physical hardware or the like. Hierarchy model in computer software development application can be implemented step by step system design abstraction, decomposing complex systems function of each part, gradual softening in the application software system design. Layered structure model to develop computer software control, scalability, better, improve the stability of computer software applications. If a layer of computer functionality changes, and maintain only the associated lower impact on the entire computer system caused by small, not related to the other levels of the system. In addition, the application of computer software development layered model that can support computer software reuse, self-developed software. In certain interface standards, each level can achieve seamless computer software and software combination.

\section{Computer Application Software Development of Stratification Technology}

Computer software development and application of technology in a double, on the one hand can enhance the efficiency of software development, on the one hand it can reduce the consumption of long-time software development. Double-layer technology including servers, clients, two endpoints, 
including servers used for receiving user information, and the user needs information Expand the appropriate induction, and then transmitted to the client; the client is more applicable for providing a user interface, at the same time put some logical relationship formed under the appropriate circumstances to start treatment. Computer application software development stratification technology, the corresponding application infrastructure, not only to ensure that the computer server with good performance, but also to ensure that the number of users can not be too much, as long as the number of users exceeds the range of computer software will produce a system error, in addition to It will make the software runs slower pace, which can not effectively meet user needs.

Double Layer is based on technology developed from the comparison with the Double-layer technology, the three-tier application server technology on the one hand to promote a certain level of improvement, on the one hand to promote the use of the computer user data storage performance strengthened. Three computer application software development technology, not only can improve the efficiency of computer access to information, but also to the promotion of effective human-computer interaction to reach a mutual information, significantly improve the efficiency of the computer. Layer with the database layer, business process layer and the interface layer mainly in which the database layer for the business process layer to expand the application range of the verification, carried out simultaneously on the corresponding database data query, get the results back via a reasonable analysis and transfer to business layer; a user interface layer is used effectively need to expand the system to collect, expanded data information via the appropriate treatment, the further transmission of information data collection results to the business layer; business process layer is used to analyze the real needs of the user, also issued corresponding request to expand the information data processing, extraction. Even three computer technology can improve the efficiency to a certain extent, but once the complex user environment, you can not on the database layer, business process layer and the interface layer reasonable division.

In computer software development, computer application technology double, triple technique were unable to effectively address the complex environment of use, so it should be the three art database layer, business process layer and the interface layer is a reasonable one by one division, so only gradually reduce mutual influence between its various levels generated, that is, should be a three-step technology developed into solid, technology, and covers the four major technology database layer, WEB layer, business layer and the storage layer.

In specific areas relevant data or computer running the background, the software developers, be sure to four technology-based structure of the database layer is divided into the integration layer, resource layer, applied to further improve the efficiency of the computer is running, and can Related equipment to be met unconventional operational requirements. At this stage, for five technology is widely used in a J2EE environment, which it launched a conventional three-tier technology further developed, covering client tier, Web presentation layer, business layer, integration layer and resource layer, five-layer technology online shopping. Wherein the client tier, Web presentation layer is extended from three technology comes to carry out running on the client is the client layer, running on the server is the Web to carry out the presentation layer; integrates data persistence layer is to carry out access environment to carry out the application, resource layer is the database layer. Select the kind of model, scientific follow their application logic will be able to produce the different components, and to this part of the component disposed on different devices.

\section{Conclusion}

Computer technology continues evolve and progress promote the development of computer software development business. Computer software development is faced with increasingly complex network environment and increasing user demand and need to continue the development of computer software application stratification technology. Stratification technology can not only improve the efficiency of computer software development, but also can reduce software development time and greatly reduces the cost of software development and therefore the application of science stratification technology meets the needs of users. 


\section{References}

[1] Xu Fuli. Stratification technology in computer software development [J]. Silicon Valley, 2013 (23)

[2] Yang Ke. Stratification technology application results in computer software development analysis [J]. Software. 2013, (10)

[3] Ye Na, Zhu Xiujuan. On the computer software development using stratification technology [J]. The wireless Internet technology, 2013, (06)

[4] Zuo Cen. Analysis of the application and development of computer software development technology prospects [J]. Intellect, 2013, (12)

[5] Wang Lihua. On the computer software development using stratification technology [J]. Henan Science and Technology. 2013, (05) 